\title{
The Tinyphoon's Control Concept
}

\author{
Gregor Novak*, Charlotte Roesener ${ }^{\dagger}$, Markus Bader*, Tobias Deutsch*, Stefan Jakubek ${ }^{\ddagger}$, \\ Stefan Krywult* and Martin Seyr ${ }^{\ddagger}$ \\ *Institute of Computer Technology, Vienna University of Technology,Vienna, Austria, \\ (novak, deutsch)@ict.tuwien.ac.at, (markus.bader, stefan.krywul)@clara.tuwien.ac.at \\ $\dagger$ Department of System Design, Faculty of Science and Technology, Keio University, Yokohama, Japan, \\ roesener@comp.sd.keio.ac.jp \\ $\ddagger$ Division of Control and Process Automation, Institute for Mechanic and Mechatronic, \\ Vienna University of Technology, Vienna, Austria, (jakubek, seyr)@impa.tuwien.ac.at
}

\begin{abstract}
Control concepts for mobile service robots are a key issue in robotic research. With the use of mobile robots in the domestic area, further challenges entails new designs facilitating efficient control concepts for fast motion with reduced computational capacity. This paper presents motion control designed for an autonomously acting two-wheel driven robot, which fits in a cuboid with $75 \times 75 \times 150 \mathrm{~mm}$. Due to the outstanding design of mechanical and electrical components, the robot is capable to perform high precision movements up to a speed of $2.5 \mathrm{~m} / \mathrm{s}$, featuring a vision unit that allows a fast environment recognition interpreting current position based on an application dependent world model. Based on predictive control algorithms, trajectory tracking and point stabilization are facilitated. The use of realtime communication for all units allows fast interaction of all components exchanging information, which is indispensable for fast motion control.
\end{abstract}

\section{INTRODUCTION}

In this paper the control concept of the Tinyphoon ${ }^{1}$ robot is described. Motion control is a main issue in robotics. Today the major contingent of robots deployed over the world is for industrial use. With new application fields in domestic area a new generation of robots has become evident. These robots face new challenges in mobility and cost efficiency in addition to industrial applications. Especially recognition and motion control systems of industrial robots, e.g. inserted for assembling processes in a factory site, are often controlled manually or by automatic control system possessing additional computational power. Therefore these robots can fulfill tasks in real-time without facing severe limitations in energy consumption, computational effort or cost. Though mobile service robots have to cope with limited energy resources, using control systems of limited computational capacity. Compared to current industrial robots, modern mobile service robots for domestic use have to act quickly in dynamically changing environment without external help. These requirements entail an improvement in robotic technology focusing on efficient object recognition and complex autonomous robotic behavior. A main issue is to integrate advanced motion control to accomplish complex action patterns.

Due to these endeavours there has been established a progressive way to match with similar researches: robot soccer. Grouped in different categories (leagues) the efficiency of new

${ }^{1}$ http: //www.tinyphoon. com technologies for future robots are evaluated. In this paper the proposed concepts have been designed for a tiny two-wheel driven robot called Tinyphoon, which preliminary generations [1] were playing soccer in the MiroSOT league. Meanwhile the robot is being advanced to a fully autonomous robot. The robot's components are distributed over several units, whereas the motion unit performs main tasks of motion control. In the following, an introduction of the Tinyphoon's system architecture with its mechanical and electrical components is given. Afterwards the software subsystems with their algorithms are described.

\section{A. Problem Analysis}

The design of a complete autonomous mobile robot of this size demands new approaches for hardware and software implementation to meet a series of challenges:

- Autonomous behavior requires "intelligence" integrated in the robot itself.

- In order to achieve highly dynamic behavior of the robot, fast processing of essential information is necessary.

- As processing power for data processing is limited, sensory data has to be reduced to a extracted set of relevant information for recognition processes.

- Low power consumption of processor hardware represents a strong limitation for all mechanical equipment, e.g. sensors and electric drive.

- The size of the system is limited to a cuboid of $75 x 75 x 150 \mathrm{~mm}$.

Facing these restrictions, no standard industrial solutions for sensors, processing unit or electrical drive can be used entailing the need for complete new robotic design from scratch.

\section{PRELIMINARY WORK}

Within the Center of Excellence for Autonomous Systems at the Vienna University of Technology ${ }^{2}$ an architecture for a tiny, fully autonomous, mobile robot, called Tinyphoon [2] was developed. The primary innovative objective achieved by the design of the tiny robot is to enable it to act completely autonomously within its environment deriving motion planing

${ }^{2}$ http: //clara.tuwien.ac.at 
without external main frame. To fulfill this requirement, generally speaking the robot is composed of distributed components based on a modular architecture, consisting of two principal units, each one dedicated to a different set of tasks. These units are: the motion unit and the vision unit interacting with the mechanical subsystem. The robot has to be capable of processing the whole data stream from its on-board sensors within itself, without the help of an external host computer. The key challenge in the design of the Tinyphoon (Fig. 1) is to design low power high performance electronics and optimized software algorithms. The time available for creating a behavioral response is extremely short so that the whole calculation process, starting with the sensory perception of the environmental data, has to be finished within a few milliseconds [3].

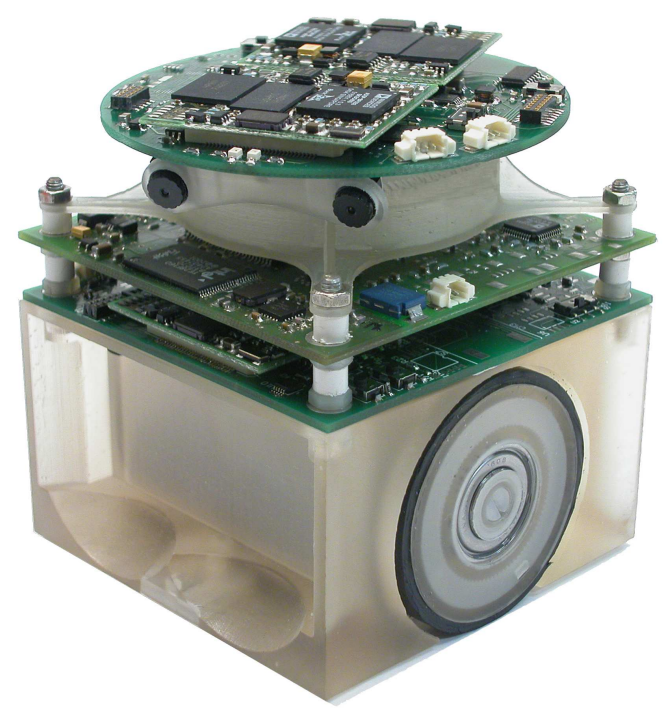

Fig. 1. Tinyphoon Robot

\section{A. Mechanical Subsystem}

The chassis of the Tinyphoon is made of fiber-reinforced plastics and houses two DC motors and a rechargeable battery. It is designed to have a very low lying center of gravity. The motors can speed up the robot to more than $2.5 \mathrm{~m} / \mathrm{s}$ with an acceleration of more than $5 \mathrm{~m} / \mathrm{s}^{2}$. Two high precision encoders with a resolution of 512 steps reduces the tolerance to $0.5 \mathrm{~cm} / \mathrm{m}$. Another outstanding feature is the rotatable head for the vision system.

\section{B. Motion Unit}

The motion unit (Fig. 2) controls the motors and is equipped with various sensors: two two-axis acceleration sensors, a gyro sensor and a magnetic field sensor. A processor from Infineon $^{3}$ 's XC series is used for collecting data from the analog sensors and for recording the movement of the wheels. The motion control is implemented using an Analog's's Blackfin processor.

\footnotetext{
${ }^{3}$ http: //www. infineon.com

${ }^{4}$ http: //analog.com
}

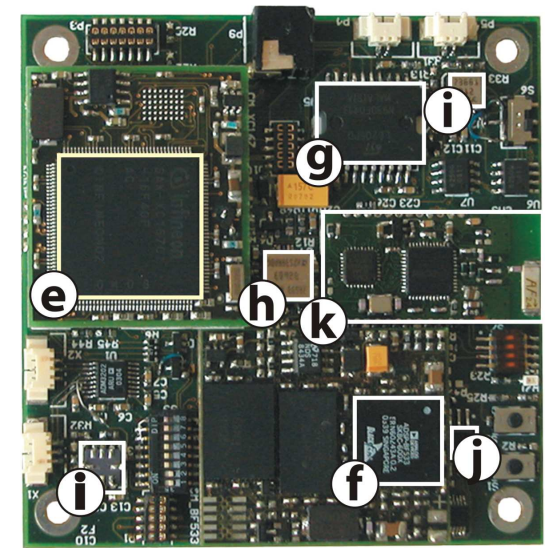

(e) Infineon XC, (f) Analog Devices Blackfin DSP, (g) Motor Driver Unit, (h) Gyro Sensor,

(i) 2x Acceleration Sensors, (j) Magnetic Field Sensor,

\section{(k) $2.4 \mathrm{GHz}$ Radio Module}

Fig. 2. Motion Unit

\section{Vision Unit}

The vision unit extends the Tinyphoon robot with stereo vision capabilities. Two CMOS cameras with a resolution of $320 \times 240$ px can be connected to the board, which is equipped with 2 Blackfin DSP Core Modules. Fig. 3 shows the hardware configuration.

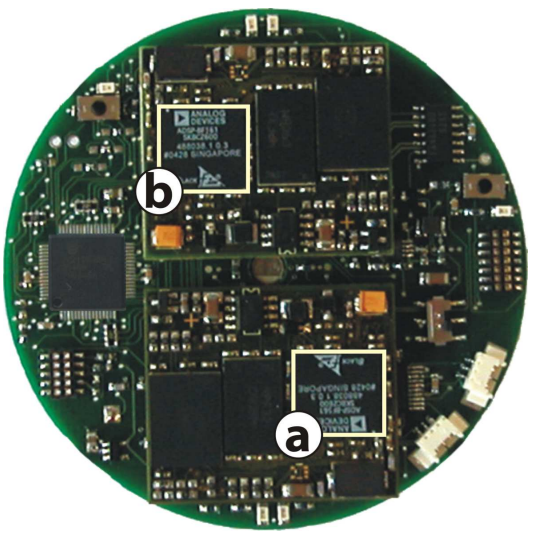

(b) Dual Core Blackfin DSP on a Core Module (b) Single Core Blackfin DSP on a Core Module

Fig. 3. Vision Unit

\section{Communication between the Units}

All units of the robot are interconnected with a real-time bus, in order to guarantee that messages arrive in time and with minimal jitter, which is required for prediction and feedback control algorithms.

\section{CONTROL CONCEPT}

The control of Tinyphoon is based on its vision system. The vision sensor generates information of the environment. 
However, for motion control the feedback from the motors' encoder, acceleration and gyro sensors are used.

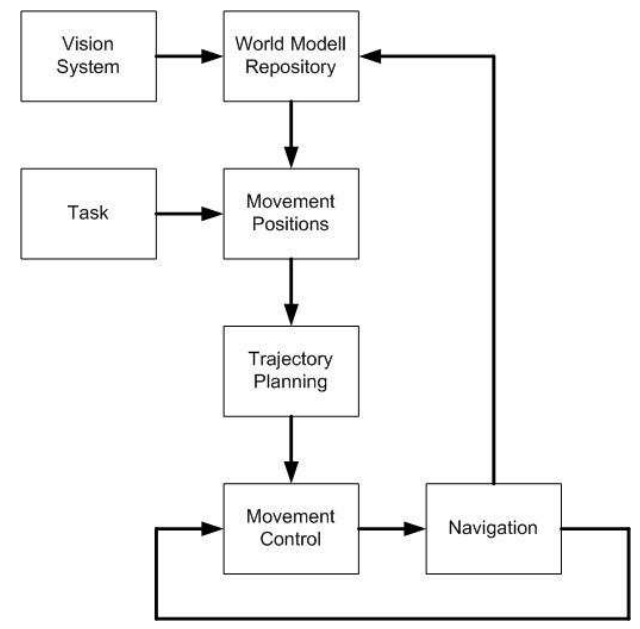

Fig. 4. Control Concept

The World Model Repository (WMR) (Fig. 4) processes the data generated by the vision system and fuses them with movement data provided by the navigation as well as with pre-existing information of the environment. The movement positions which are necessary for the trajectory planning are calculated based on specified tasks and the view of the world provided by the WMR. The movement control direct the robot to its given target using the motion data from the navigation.

\section{VISION SYSTEM}

The vision system integrated in Tinyphoon allows the robot to detect edges, lines, spheres and plane rectangles in an 3D space. Spheres and rectangles are handled as objects and must be predefined in an object model. The vision system can localize objects relative to the position of the robot. Objects are identified in the image plane first by their color. An exact detection will be applied exclusively in the local area around the corresponding color blob. A shape model extracted with the aid of the object model represents the 2D projection of objects. Based on this shape model the search algorithm for a local area of a blob is selected to determine the correspondence between the blob and an object. Similar to [4] the dataflow is used to rate detected objects so that an accuracy value can give information how appropriate the detection was. Fig. 5 shows the flow-chart of the program used in the vision system. The known weakness of this approach, which has been reported in the majority of similar hardware designs e.g. [5], [6] and [7] is the limited L1 cache of the used controllers. This problem can be avoided by an extensive use of the DMA controller to hold only the necessary data in the L1 cache. The used Analog Devices Blackfin BF561 DSP is a dual core digital signal processor which allows to process two captured images of the stereo system at the same time. An edge depth map builds the base to extract 3D lines by using a line algorithm similar to [8]. A manual calibration of the exposure time, black/white balance and other camera settings is essential that both images are representing the same color for one object. The dynamic change of the camera resolution and a smaller search window in the image allows to system to work at least with a frequency between 5 and $20 \mathrm{~Hz}$.

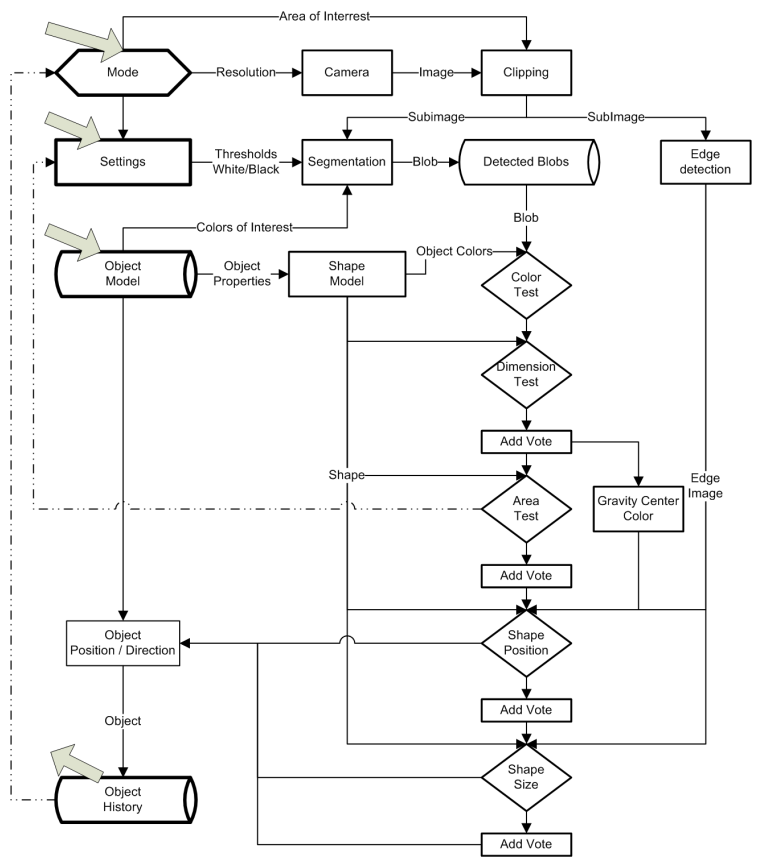

Fig. 5. Data flow of the object detection. The large arrows mark the in- and output.

\section{WORLD MODEL REPOSITORY}

The World Model Repository (WMR) serves as an interface between the sensor data providing units (vision and motion) and the movement position calculation. As described in Section IV the vision unit provides relative position of predefined colored geometric objects like an orange golf ball. From the motion unit odometric data from the internal sensors is provided. Based upon the data from the WMR a new target is calculated. Afterwards the designated movement is transmitted to the motion unit and as feedback to the WMR.

Data provided by the WMR adapted for the application field of robot soccer can be divided into two categories:

- Static data: Static data do not change during the game consisting of the shape and coloring of the playground, the ball, the teammates and the opponents. Also information like maximum speed, number of robots per team belongs to this category.

- Dynamic data: As the ball and all robots change their position during the game, they have to be estimated and predicted using sensor data and estimation algorithms like Kalman filter [9] or particle filter. The position contains the axis $x, y$, the heading $\varphi$ and the quality of the position estimation $q$.

In case the shape of the opponents is not known in advance, the data have to be handled as dynamic data, too. 


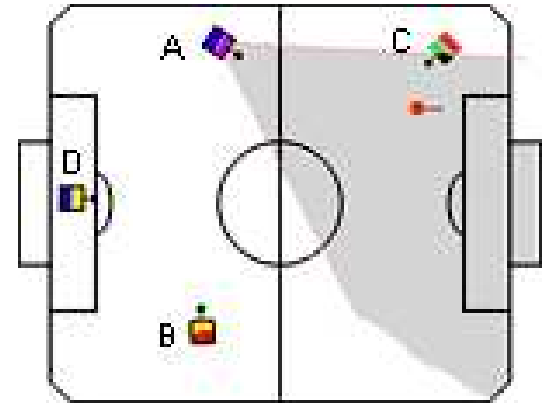

Fig. 6. Situation on the Playground

Fig. 6 shows a typical situation in robot soccer. The robot A in the upper left section perceives via vision sensor information about the ball position, the position of one of the opponents (C) ${ }^{5}$ and the opponent goal. Obviously no current information about the two robots B and D outside the visual field is available. Using the odometric sensor data and the relative position of the visible goal the self localization is performed. Furthermore the ball position and the positions of visible robots are estimated. If a robot gets out of the vision field, the uncertainty of the position estimation increases vastly with time. Thus the position of robots with no information update for several estimation rounds are treated as unknown. Unlike robots, the ball has a linear movement, which allows a position estimation and prediction even without new sensor information. Because of this and the fact that the ball is the most important object of a unique shape, the ball position will be estimated at any time.

As mentioned before, there are three different types of localization: 1.self localization, 2. ball localization, and 3 . robot localization. The self localization is realized by means of a particle filter integrating the external sensed position data and the internal motion update measured by the odometry. To predict the linear moving ball, a combination of the Kalman filter with a direction change utility calculating the reflexion of the ball on a border during following the predicted path. Observed robots are processed in singular observations. Even if a robot is visible in two consecutive rounds, these observations will be handled independently. Due to the limited sensory data in combination with the non linear movement of robots the localization of other robots is hardly feasible.

The generated dynamic position data is stored in position vectors whereas the a priori knowledge about the shape of the robots and the playground is stored using geometric representations. In order to determine the validity of a positionwhich means in case of a soccer game it must be inside the borders of the playground - a grid cell decomposition is performed. Each cell has a size of e.g. $5 \times 5 \mathrm{~cm}^{2}$ and its value represents the believe that the position is inside. Due to the uncertainty of position estimations the probability does not drop from 1 to 0 at the borders. The value starts fading at the

\footnotetext{
${ }^{5}$ The position information quality of the opponent robot may be reduced due to the fact, that it is only partially visible.
}

closest position to the borders where it is for sure within the borders. 0 will be reached at the opposite point outside of the playground. Fig. 7 shows such a grid where black represents 1 and white $0^{6}$, the levels of gray represent a value between 0 and 1.

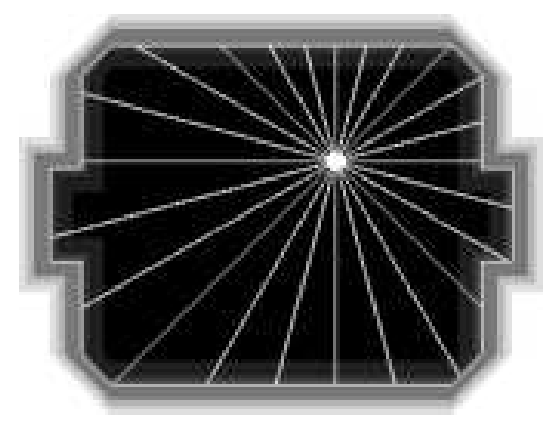

Fig. 7. Precalculated map for distances to the boards and the general believe that the position is inside the playground

The white lines which convergent in one point represent another precalculated value - the distances from a position to the borders at various directions. These distances are calculated for every cell. Using a distance sensor and a particle localization, the quality of the self localization can be increased [10].

To enhance reliability and availability of position data, information exchange among the teammates may be introduced.

\section{COMMUNICATION}

The Tinyphoon is equipped with a communication system that interconnects all units. Since the robot acts autonomously in a very dynamic environment gathered information is only valid for a very short time. A real-time communication system is proposed for transferring all necessary information timely and with a minimal jitter. It has to fit the needs of such a deeply embedded system like the Tinyphoon. Fault tolerance is currently not considered. But a malfunction of the communication system can be detected and a transition to a fail-save state can be initiated.

\section{A. Importance of Real-Time Communication}

In the automotive and avionics industry the application of real-time communication has become as a matter of course over the last years. The growing complexity of embedded systems leads to modular and distributed design, which allow considering each subsystem on its own to increase the flexibility and the reusability of the system.

Timing requirements do not only concern each single subsystem but also the communication system providing the interconnection. Traditionally, communication is performed by means of event-triggers in deeply embedded systems like the Tinyphoon. If an event happens, a data packet will be sent to inform the other subsystems. The timeliness of this communication cannot be guaranteed because it depends on the cooperation of every subsystem. Even if a mechanism for

\footnotetext{
${ }^{6}$ The white border in the middle of the fade represents the real playground and is drawn for visualization purposes
} 
prioritizing packets is implemented like in CAN [11], communication timings (e.g. meeting the deadlines and restraining the jitter) cannot be assured. The occurrence of too many events in a period of time inhibits the communication completely. Hence, the behavior of the subsystems is only defined in the functional domain but it remains undefined in the temporal domain and the system lacks temporal composability [12]. Therefore, a real-time communication system is necessary to allow a global scheduling of the communication.

\section{B. Benefits of Real-time Communication}

The modular design of the subsystems in both, the value and the time domain guarantees the derivation of full functionality of communication when enhancing the architecture adding a new subsystem. Moreover a unit can be exchanged by another, that performs the same service in a different way without revalidating the communication.

The assignment of data packets to a specific time slot can be used as an implicit time stamp that is valid throughout the cluster. A global time base can be established for synchronizing all subsystems (e.g. the current state of the motion unit can be recorded at the same moment as the vision unit captures an image). Fusing this synchronized data is much simpler than fusing data collected in different points in time.

The real-time communication system guarantees a limit of the latency and therefore confines the reaction time and makes it predictable. Moreover, the low jitter of the real-time communication makes feedback control algorithms stable. The majority of algorithms can only deal with a fixed latency.

\section{Predictive Trajectory Tracking AND PROPRIOCEPTIVE NAVIGATION}

For Tinyphoon a predictive control algorithm is used, which enables both trajectory tracking and point stabilization [13]. This algorithm is deployed in conjunction with a proprioceptive navigation algorithm, as depicted in Fig. 8.

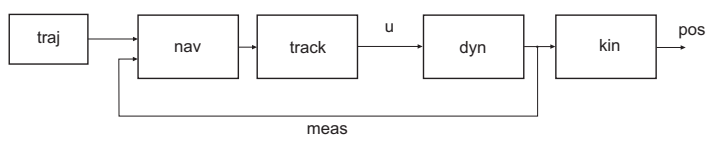

Fig. 8. Control Algorithm

\section{A. Navigation}

Navigation using either inertial or odometric data for posture estimation is known as proprioceptive navigation or dead reckoning as opposed to exteroceptive navigation [14]. Naturally, the position error is subject to unbounded accumulation. Nevertheless, improved proprioceptive navigation systems help increase the allowable travel distance between absolute position updates [15].

The standard method for sensor fusion is extended Kalman filtering (EKF), e.g. [16], [17]. An EKF yields an optimal estimate in the sense that the statistical variance of the estimated states is minimised. However, biased measurement produces erroneous estimates. Typical sources of measurement bias are inevitable sensor drift for inertial sensors and inaccuracies of geometric parameters and wheel slip for odometric measurement.

To overcome the effects of wheel slip or bumps (causing discontinuous ground contact), which introduces a non-systematic error into odometry, inertial data is used only transiently during periods where odometric measurements seem to be unreliable. Aside from these periods, odometric measurement is used exclusively, because it is not affected by drift and has little bias when properly calibrated, [15], [18]. The novelty in the used approach is the usage of both gyro and acceleration sensors to transiently substitute for wheel encoder data when necessary, thus enabling reliable side-slip angle estimation and tangential slip detection and ultimately allowing for slip control [19].

\section{B. Trajectory Tracking Control}

The kinematics of the unicycle-type mobile robot are a classical example of a nonholonomic nonlinear control system, also called nonholonomic integrator (NHI), [20]. It was first shown by Brockett in [21], that this type of system cannot be stabilised by continuous, time-invariant feedback, although it is controllable in a nonlinear sense. Furthermore, it can be shown using a methodology by Isidori in [22], that the NHI cannot be feedback-linearised.

Among the major strategies for trajectory tracking and path following control are approaches making use of dynamic posture error models [23], [24], concepts involving integrator backstepping [25], [26] and dynamic feedback linearisation [27], [28]. Most control schemes (including a discontinuous scheme in [29]) designed for trajectory tracking suffer from singularities or loss of controllability at zero reference velocity, i. e. they are incapable of compensating residual posture errors after the reference trajectory reaches a stationary point.

Posture stabilization poses a much more difficult problem. The various approaches trying to circumvent the restrictions pointed out in [21] include sliding mode control or similar discontinuous control laws [30], [31], time-varying controls [32], hybrid control laws employing logic-based switching [33] and concepts making use of invariant manifolds [34].

Posture stabilisation does not necessarily involve a preplanned trajectory. As a consequence, it is impossible to influence the way how the target posture is reached and therefore obstacles can not be taken into account.

According to [28] "the necessity of using two different control laws for trajectory tracking and posture stabilization" is evident illuminating the problem. Our novel approach is employing numerical optimization of the control inputs rather than any explicit feedback control law. This solves both the trajectory tracking and the posture stabilization problem. As another feature of the proposed scheme a possible side-slip angle (as estimated by the navigation part of the algorithm) is taken into account [35].

\section{CONCLUSION}

This paper shows efficient control concepts for a mobile robot coping limited in size, power consumption, and 
processing capacity. Field-in tests on the play ground and performance evaluation have verified the simulated results (Fig. 9). Within these simulations correct obstacle avoidance and trajectory tracking control has been evaluated. Showing the high efficiency of real-time object recognition in order of few milliseconds suggests the capability to operate in highly dynamic environments. With the implementation of a modular design splitting the components into diverse distributed units interconnected by means of real-time communication, allows the reuse and separated enhancement of all subsystems. Although the first application field has been robot soccer, the majority of methods can be adapted for all kinds of autonomous mobile robots in several application fields.

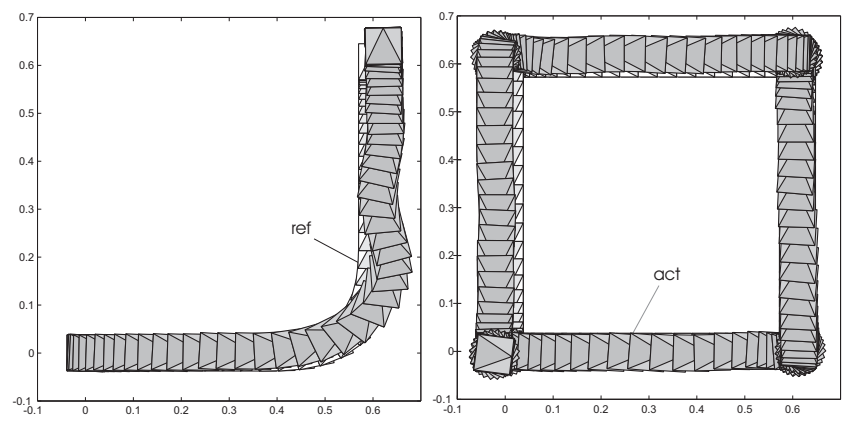

Fig. 9. Reference and actual trajectories (obtained at 24.4 frames per second by video measurement) for corner (left) and square (right)

\section{ACKNOWLEDGMENT}

This paper was written within the Center of Excellence for Autonomous Systems of the Vienna University of Technology (CEAS).

\section{REFERENCES}

[1] G. Novak. Roby-go, a prototype for cooperating mirosot soccer-playing robots. Journal of Advanced Computational Intelligence and Intelligent Informatics (JACIII), Volume 9, Numbers 4:409-414, July 2005.

[2] G. Novak and S. Mahlknecht. Tinyphoon - a tiny autonomous mobile robot. In IEEE ISIE 2005 International Symposium on Industrial Electronics 2005, pages 1533-1538, Dubrovnik, Croatia, June 20-23 2005.

[3] Mahlknecht, S., Oberhammer, R. and Novak, G. A real-time image recognition system for tiny autonomous mobile robots. Real-Time Systems, Volume 29, Numbers 2-3.

[4] M. Bader, M. Albero, R. Sablatnig, J. E. Simó, G. Benet, G. Novak, and F. Blanes. Embedded real-time ball detection unit for the yabiro biped robot. WISES'06, Fourth Workshop on Intelligent Solutions in Embedded Systems, 4, 2006.

[5] A. Rowe, C. Rosenberg, and I. Nourbakhsh. A low cost embedded color vision system. In Proceedings of IROS 2002, 2002.

[6] S. Mahlknecht, R. Oberhammer, and G. Novak. A real-time image recognition system for tiny autonomous mobile robots. In IEEE RealTime and Embedded Technology and Applications Symposium, pages 324-330, 2004.

[7] G. Novak, A. Bais, and S. Mahlknecht. Simple stereo vision system for real-time object recognition for an autonomous mobile robot. In IEEE International Conference on Computational Cybernetics (ICCC2004), pages 213 - 216, 2004.

[8] H. Entner. Real-time 3d reconstruction for autonomous football playing robots using a feature based stereo approach. Master's thesis, Vienna Universtiy of Technology, January 2005.

[9] Emil Kalman, Rudolph. A new approach to linear filtering and prediction problems. Transactions of the ASME-Journal of Basic Engineering, 82(Series D):35-45, 1960.
[10] Dieter Fox. Markov Localization: A Probabilistic Framework for Mobile Robot Localization and Navigation. PhD thesis, Institute of Computer Science III, University of Bonn, Germany, 1998.

[11] Robert Bosch GmbH. CAN specification, version 2.0, 1991.

[12] Kopetz. Real-time systems: design principles for distributed embedded applications. Kluwer Academic Publishers, 1997.

[13] C. Canudas de Wit, H. Khennouf, C. Samson, and O. J. Srdalen. The Book of Mobile Robots, Nonlinear Control Design of Mobile Robots. World Scientific, Singapore, 1996.

[14] E. Fabrizi, G. Oriolo, S. Panzieri, and G. Ulivi. Enhanced Uncertainty Modeling for Robot Localization. In Int. Symp. on Robotics with Application (ISORA'98), volume 7, pages 313-320, 1998.

[15] J. Borenstein and L. Feng. Gyrodometry: A new method for combining data from gyros and odometry in mobile robots. In IEEE International Conference on Robotics and Automation, Minneapolis, Minnesota, 2005.

[16] S.I. Roumeliotis and G.A. Bekey. An Extended Kalman Filter for frequent local and infrequent global sensor data fusion. In SPIE International Symposium on Intelligent Systems and Advanced Manufacturing, 1997.

[17] Y. Fuke and E. Krotkov. Dead reckoning for a lunar rover on uneven terrain. In IEEE International Conference on Robotics and Automation, Minneapolis, Minnesota, 1996.

[18] R. Mazl and L. Preucil. Sensor data fusion for inertial navigation of trains in GPS-dark areas. Intelligent Vehicles Symposium, 2003. Proceedings. IEEE, pages 345 - 350, June 2003.

[19] M. Seyr and S. Jakubek. Proprioceptive navigation, slip estimation and slip control for autonomous wheeled mobile robots. In IEEE RAM, Bangkok, Thailand, 2006.

[20] J. P. Hespanha and A. S. Morse. Stabilization of Nonholonomic Integrators via Logic-Based Switching. Automatica's Special Issue on Hybrid Systems, to appear., 1998.

[21] R. W. Brockett. Differential Geometric Control Theory, chapter Asymptotic Stability and Feedback Stabilization. Birkhauser, 1983.

[22] A. Isidori. Nonlinear Control Systems. Springer, 1989.

[23] R. Fierro and F.L. Lewis. Control of a Nonholonomic Mobile Robot: Backstepping Kinematics into Dynamics. Journal of Robotic Systems, 14(3):149-163, 1997.

[24] T.-C. Lee, K.-T. Song, C.-H. Lee, and C.-C. Teng. Tracking Control of Unicycle-Modeled Mobile Robots Using a Saturation Feedback Controller. IEEE transactions on Control Systems Technology, 9(2):305317, Mar. 2001.

[25] Z.-P. Jiang and H. Nijmeijer. Tracking Control of Mobile Robots: A Case Study in Backstepping. Automatica, 33(7):1393-1399, 1997.

[26] W. Wu, H. Chen, and Y. Wang. Backstepping Design for Path Tracking of Mobile Robots. In IEEE International Conference on Intelligent Robots and Systems, volume 3, pages 1822-1827, Oct. 1999.

[27] B. d'Andrea Novel, G. Campion, and G. Bastin. Control of Nonholonomic Wheeled Mobile Robots by State Feedback Linearization. The International Journal of Robotics Research, 14(6):543-559, Dec. 1995.

[28] G. Oriolo, A. De Luca, and M. Vendittelli. WMR Control Via Dynamic Feedback Linearization: Design, Implementation, and Experimental Validation. IEEE Transactions on Control Systems Technology, 10(6):835851, Nov. 2002.

[29] C. Samson and K. Ait-Abderrahim. Feedback Control of a Nonholonomic Wheeled Cart in Cartesian Space. IEEE International Conference on Robotics and Automation, Sacramento, CA, pages 1136-1141, Apr. 1991.

[30] A. Bloch and S. Drakunov. Stabilization of a Nonholonomic System via Sliding Modes. IEEE Conference on Decision and Control, 1994.

[31] J.-M. Yang, I.-H. Choi, and J.-H. Kim. Sliding Mode Control of a Nonholonomic Wheeled Mobile Robot for Trajectory Tracking. IEEE International Conference on Robotics and Automation, 4:2983-2988, May 1998.

[32] C. Samson. Control of Chained Systems. Application to Path Following and Time-Varying Point-Stabilization of Mobile Robots. IEEE Transactions on Automatic Control, 40(1):64-77, Jan. 1995.

[33] A. P. Aguiar and A. Pascoal. Stabilization of the Extended Nonholonomic Double Integrator via Logic-Based Hybrid Control. SYROCO'OO - 6th IFAC Symposium on Robot Control, 2000.

[34] J. Luo and P. Tsiotras. Exponentially Convergent Control Laws for Nonholonomic Systems in Power Form. Systems and Control Letters, 35:87-95, 1998.

[35] M. Seyr and S. Jakubek. Mobile robot predictive trajectory tracking. In ICINCO, Barcelona, Spain, 2005. 